\title{
Application of Morlet Transform Wavelet in the Detection of Paradoxical Splitting of the Second Heart Sound
}

\author{
HM Mgdob, JN Torry, R Vincent, B Al-Naami \\ University of Sussex, Brighton, UK
}

\begin{abstract}
This study aims at utilising wavelet transforms, namely the Morlet wavelet, in early detection of a reverse in the appearance of $A 2$ and $P 2$ within the second heart sound.

With the collaboration of the Cardiology Department at Brighton and Sussex University Hospital in England, and using a newly build data acquisition system developed at the University of Sussex, a number of patients with LBBB (Left Bundle Branch Block), and others with fitted pacemakers, were studied. The data was then analysed with Morlet algorithm. From the displayed results it is relatively easy to detect the reverse in $A 2$ and $P 2$. This technique offers an effective, economic method of detecting the paradoxical splitting of $S 2$, which is otherwise very difficult to detect with the human ear. The technique can works together with the $E C G$ to increase the reliability of the diagnosis of $L B B B$ cases.
\end{abstract}

\section{Introduction}

The first heart sound has a frequency of between 25$45 \mathrm{~Hz}$, and lasting for 0.15 seconds, where the second heart sound has a frequency of about $50 \mathrm{~Hz}$, and lasts for about 0.12 seconds [1]. Both comprise of two components $\mathrm{M} 1, \mathrm{~T} 1$, and $\mathrm{A} 2, \mathrm{P} 2$ respectively. The analysis in this paper centres on the second heart sound (S2) in terms of characteristics and components.

The separation of the components in S2 is a normal phenomenon in healthy individuals during held inspiration. If this splitting persists or appears in other parts of the breathing cycle, it can indicate the presence of pathological heart conditions. However, when the separation of A2 and P2 is reversed (paradoxical splitting) it indicates the certain presence of a heart condition. One of these pathological conditions is LBBB. Which is usually seen in dilated cardiomyopathy, hypertrophic cardiomyopathy, hypertension, aortic valve disease, coronary artery disease, and a variety of other cardiac conditions.

The paper looks at patients with LBBB and pacemaker patients, as they come under the list of patients with possible paradoxical splitting of S2.

\subsection{Paradoxical splitting of $\mathrm{S} 2$}

Paradoxical splitting occurs when the closure of the aortic valve is delayed, so that the pulmonic closure occurs first followed by aortic closure. In this case the interval between pulmonic and aortic closures is heard during expiration and disappears during inspiration. One of these pathological conditions is LBBB. There are other diseases that cause paradoxical splitting, such as (i) Right Ventricular Ectopic and Paced Beats, where the delay in the left ventricular contraction resulting from ectopic or paced right ventricular beats causes the paradoxical split, (ii) Left Ventricular Outflow Tract Obstruction, which is caused by prolonged left ventricular ejection period that is usually secondary to congenital valvular aortic stenosis, (iii) WolffParkinson-White Syndrome, where there is an additional or accessory conducting pathway which leads from the atria to the ventricles, and if these pathways favour an earlier conduction of the right ventricle, this causes $\mathrm{P} 2$ to move before A2.

\section{Wavelet representation}

Due to the importance of S2 as an indication of the presence of heart disease, Tilkian [3] wrote in his book about auscultation "It is important to persist in training the ear because once you can hear the splitting of S2, cardiac auscultation becomes clinically more useful, specially detecting expiratory splitting of S2, a helpful clue identifying cardiac disease". Thus, newer investigations and developments if new techniques to make it easier to listen, analyse, and interpret the components of the second heart sound is important.

The Continuous Wavelet Transform CWT, is frequently used [4-8] in the analysis of various heart sounds and murmurs. From these studies, it was confirmed that Morlet wavelet was the best suited for the analysis of heart sounds. From the natural shape of the mother Morlet wavelet, the Morlet waveform best resembles the shape of the PCG signal of the heart sound in terms of the fundamental frequency constituents. 


\subsection{Selection of scales}

In wavelet analysis, the selection of scales is an important part in specifying the frequencies for which the mother wavelet is convoluted with the original signal. The relationship between scale and frequency is covered by the equation below:

$$
S \propto 1 / f
$$

where $\mathrm{S}$ is scale, and $f$ is the frequency equivalent to the scale for the particular wavelet, Morlet in this case.

By using Matlab ${ }^{\mathrm{TM}}$ function SCAL2FRQ, it is possible to calculate the scales corresponding to the desired frequency, which in this case is between 20 and $100 \mathrm{~Hz}$.

The syntax for this function is as follows:

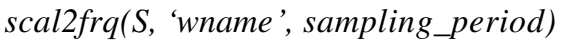

where $S$ is the scale for which the frequency equivalent is calculated, 'wname' is the name of the wavelet, and the sampling_period is (1/sampling frequency). This function starts by finding the central frequency of the Morlet wavelet, then finds the pseudo frequency for the mother wavelet depending on the sampling period.

It was found that scales from 30 to 160 cover the frequency band of interest, corresponding to frequencies ranging from $100 \mathrm{~Hz}$ to $20 \mathrm{~Hz}$.

Tests were conducted to determine the step size at which the scale-to-frequency is calculated, and it was found that a step of 2 gives satisfactory resolution compared to a step of 1 with less processing needed to find the coefficients.

\subsection{Morlet analysis of normal individuals}

Before applying the CWT algorithm, the data is filtered with a band-pass Butterworth filter to eliminate noise and components below $20 \mathrm{~Hz}$ and above $200 \mathrm{~Hz}$. This is achieved in Matlab with the following commands:

$$
\begin{gathered}
{[a, b]=\operatorname{butter}\left(2,\left[\begin{array}{ll}
20 & 200]
\end{array} * 2 / f s\right) ;\right.} \\
D=\operatorname{filtfilt}(a, b, D) ;
\end{gathered}
$$

Where $f_{s}$ is the sampling frequency, $\mathbf{D}$ is the data variable, and $a, b$ are the filter coefficients.

After the filtering stage, the Morlet algorithm is applied to the data using the scales, which were calculated. Below is the command line to generate the convolution coefficient between the Morlet wavelet and the data at various scale values:

$$
\operatorname{coefs}=\operatorname{cwt}\left(D,[30: 2: 160], \text { morl }^{\prime}\right) \text {; }
$$

The generated coefficients are then scaled further, and plotted as a contour plot, using the Matlab contour function.

$$
\text { contour(Time,freq,coefs); }
$$

A sample of PCG signal for S2 and the plot of the resulting contour after the analysis with the CWT algorithm are illustrated in the figure 1 .
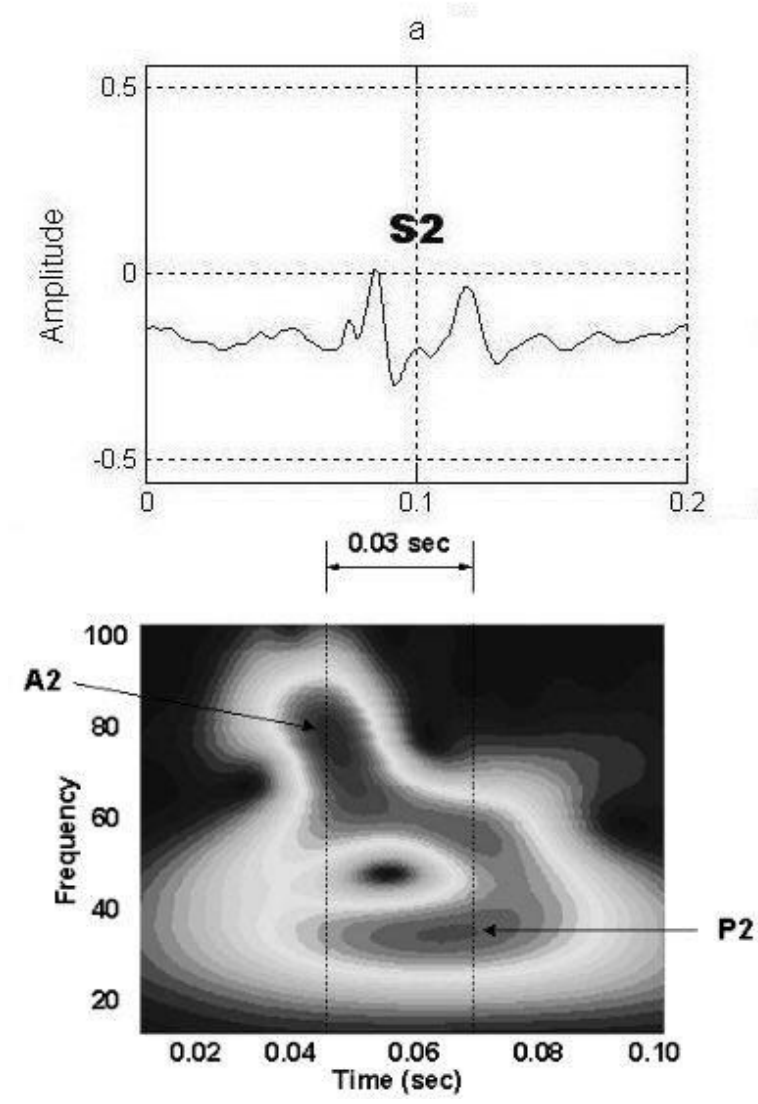

Figure 1. (a) Isolated PCG signal of second heart sound, (b) corresponding contour plot showing both components of S2. It can be seen that A2 appears before $\mathrm{P} 2$ in normal individuals

\section{Study of data collected from patients with heart disease}

In this section, data collected recently (February to June 2003) is analysed. The data was acquired in the outpatient department of the Sussex and Brighton University Hospital.

In total 22 patients with a variety of heart conditions were recorded, of which, 5 are patients with $\mathrm{LBBB}$, and 10 are patients with a pacemaker fitted. The reminder have other conditions. All the recordings were taken with patients in the semi-supine position, and with pulse oximeter and ECG signals recorded for synchronising purposes, and possible future studies of the relations between the three signals.

\subsection{Results of $\mathrm{LBBB}$ patients}

Bundle branch block is when the conduction of electrical pulses from the atria to the ventricles are impeded causing what is called intraventricular 
conduction defects (IVCD). The left bundle carries nerve impulses that cause contraction of the left ventricle. There are several types of $\mathrm{LBBB}$, each has its own characteristic mechanism of failure. In each case, the nerve impulses are blocked or delayed. Patients with LBBB almost always have underlying heart disease, such as arteriosclerosis, congenital heart disease, myocarditis, myocardial infraction, and hypertrophic cardiomyopathy [2]. ECG tests are usually carried out to detect suspected LBBB.

Below are 5 plots taken from the five patients under study, where A2 can evidently be seen lagging behind $\mathrm{P} 2$. The frequency range of $\mathrm{P} 2$ lies between 20 and 60 $\mathrm{Hz}$, and the range for the aortic valve closure lies between 50 and $90 \mathrm{~Hz}$.

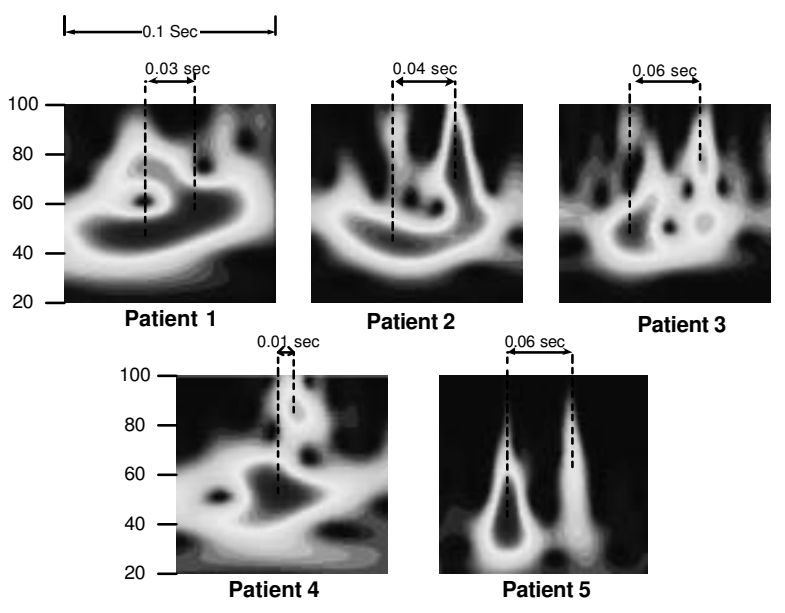

Figure 2. Plots of 5 LBBB patients with delayed A2 in relation to $\mathrm{P} 2$.

The change in the characteristics of the plot, is a reflection of a number of factors, such as:

1. The split periods highlighted in the plots vary depending on the severity of the LBBB, and the degree to which the heart muscles conducting the electrical pulses are damaged.

2. The change of the heart dynamics, due to dilation of the right ventricle, resulting from conditions such as hypertrophic cardiomyopathy, changes the frequency of S2 and P2.

As a result of the above, the paradoxical splitting is not expected to appear in every expiration period. The table below lists the LBBB patients and the number of times the paradoxical splitting was apparent during a 10 second recording of each patient.

Table 1 shows that in each case of LBBB, paradoxical splitting can be detected at least once. In $60 \%$ of the patients, it was detected twice in the 10second period, and $20 \%$ for both four and one paradoxical split detection. As was mentioned in the previous section, these variations depend on severity of the LBBB and how much the dynamics of the heart are changed. Also, the results depend on how deep the breaths are during respirations
Table 1. Number of Paradoxical Splitting Detected and Split Distances (ms) in LBBB Patients

\begin{tabular}{ccrrr}
\hline PATIENT & PARADOX. SPLIT & $0-20$ & $20-40$ & $40-60$ \\
\hline 1 & 2 & 0 & 1 & 1 \\
2 & 2 & 0 & 0 & 2 \\
3 & 1 & 0 & 0 & 1 \\
4 & 4 & 1 & 1 & 2 \\
5 & 2 & 1 & 0 & 1 \\
\hline
\end{tabular}

The distance was measured from the peak of the contour plots, and converted to time in milliseconds. The human ear can distinguish between two sounds with a separation of down to $20 \mathrm{~ms}$ [10]. Even though, this can vary from an individual to another.

The table confirms that in $18 \%$ of the patients the paradoxical splitting is not detected and sound as a fusion of A2 and P2. The second band $(20-40 \mathrm{~ms})$, is a border line, meaning that for the trained ear the split in $18 \%$ of patients is detected, but could be difficult to detect to the untrained ear. Finally, in $64 \%$ of the patients, the paradoxical split is wide enough to be detected clearly.

\subsection{Results of pacemaker patients}

Patients with pacemaker implants were investigated because from the nature of the function of pacemakers, they can give similar characteristics of LBBB patients. In single and dual chamber pacemakers, a probe is usually inserted in the right ventricle, to stimulate it. This results in the right ventricle contracting before the left ventricle, which has to wait for the electrical pulse to reach it from the right side of the heart. The delay of the left ventricular contraction causes pulmonic valve to close before the aortic valve, resulting in P2 appearing ahead of A2.

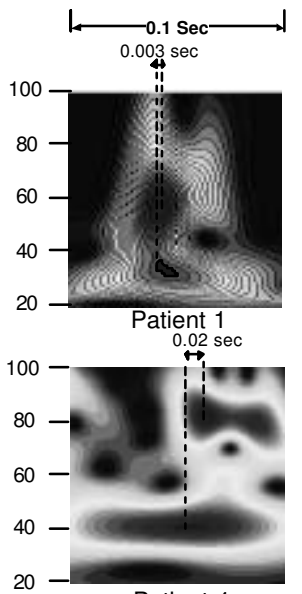

Patient 4
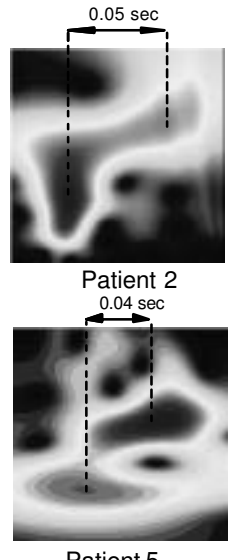

Patient 5

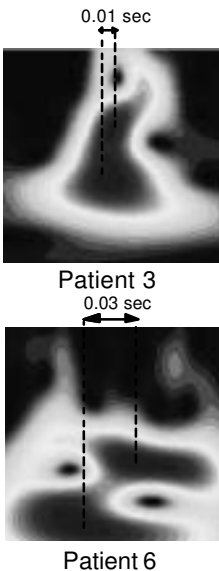

Patient 6
Figure 3. Plot of 6 patients with signs of paradoxical splitting of S2. 
The data from the 8 patients with pacemaker is statistically analysed and is presented in table below.

Table 2. Number of Paradoxical Splitting Detected and Split Distances (ms) in pacemaker patients

\begin{tabular}{ccrrr}
\hline PATIENT PARADOX. SPLIT & $0-20$ & $20-40$ & $40-60$ \\
\hline 1 & 1 & 1 & 0 & 0 \\
2 & 4 & 2 & 0 & 2 \\
3 & 3 & 2 & 1 & 0 \\
4 & 2 & 1 & 1 & 0 \\
5 & 1 & 1 & 0 & 0 \\
6 & 5 & 0 & 1 & 4 \\
7 & 0 & 0 & 0 & 0 \\
8 & 0 & 0 & 0 & 0 \\
\hline
\end{tabular}

The table shows that paradoxical splitting could be detected in 6 out of 8 patients, which constitutes $75 \%$ of the patients analysed. In $25 \%$ of the patients, the split was detected once, and in the $50 \%$ of the remaining patients, paradox splitting was detected equally with 2 to 5 times. The table also gives an idea about the audibility of each split, with splits of between $0-20 \mathrm{~ms}$ impossible to hear, $20-40 \mathrm{~ms}$ audible with difficulty, and $40-60 \mathrm{~ms}$ as easy to hear splits [9]. The table indicates that from the detected splits $44 \%$ are impossible to hear with the human ear and would be classified as a fused S2 by cardiologists. In $19 \%$ of the detected splits, the separation was between $40-60 \mathrm{~ms}$, making them audible with difficulty, and in $37 \%$ of the patients the split can be heard with a degree of ease, since the separation is higher than $40 \mathrm{~ms}$.

\section{Conclusion}

In this paper an attempt has been made to identify patients with paradoxical splitting of the second heart sound. Distinguishing such a patient, from a patient with a normal splitting of the $\mathrm{S} 2$ is a very important factor in identifying a number of dangerous heart diseases. The danger lies in that most of patients with paradoxical splitting are not aware of having any heart disease, in the early stages at least, where a clot might be developing which might lead to a heart attack or a stroke.

The technique has been applied to a number of patients with $\mathrm{LBBB}$, and found that in all these patients, it was possible to detect the presence of paradoxical splitting at least once. The analysis was also applied to pacemaker patients, and showed that it was possible to detect the split in $75 \%$ of the patients.
The technique shows a clear advantage in patients, where splitting period is below $20 \mathrm{~ms}$, which is the borderline for the human ear sensitivity. This constituted $18 \%$ of detected paradox in LBBB patients, and $44 \%$ of detected paradox in pacemaker patients.

\section{Acknowledgement}

The efforts of Prof. R. Vincent and Ms. S. Baker in the outpatient department and pacemaker clinic at Brighton and Sussex University Hospital are gratefully acknowledged for their role in acquiring patients for this study.

\section{References}

[1] Leatham A. Ausculatation of the heart and phonocardiography. second ed: Longman Group Limited; 1975.

[2] Swanton RH. Cardiology, Blackwell Science Limited 1998.

[3] Tilkian G. Understanding heart sounds and murmurs: with the introduction to lung sounds. WB Sounders Company 1993.

[4] Khadra L, El-Asir B, Mawagdeh S. The wavelet transform and its applications to phonocardiogram signal analysis. Med. Inform 1991; 16(3): 271-277.

[5] Bulgrin $\mathbb{R}$, Thompson CR, Moody JM. Comparison of short-time Fourier, wavelet and time-domain analysis of intracardiac sounds. Biomed Sci Instrum 1993; 29: 465472.

[6] Obaidat MS. Phonocardiogram signal analysis: techniques and performance comparison. J Med Eng Technol 1993; 17(6): 221-7.

[7] Bentley PM. Wavelet Transforms: An Introduction. Electronics and Communications Engineering Journal 1994; 175-186.

[8] Balster D, Chan D, et al. Digital acoustic analysis of precordial innocent versus ventricular septal defect murmurs in children. Am J Cardiol 1997; 79(11):1552-5.

[9] Hugonnet C, Walder P. Stereophonic sound recording: theory and practice. John Wiley \& Sons Ltd. 1998.

Address of correspondence

Dr. John Torry

Department of Engineering and Design

University of Sussex

Brighton, UK.

j.n.torry@sussex.ac.uk 